\title{
COMPREHENSIVE FAMILY OF UNIFORMLY ANALYTIC FUNCTIONS
}

\author{
B. A. FRASIN
}

\begin{abstract}
We introduce the subclass $\mathcal{U}_{\mathcal{T}}(\Phi, \Psi ; \alpha, \beta)$ of analytic functions with negative coefficients. Coefficient inequalities, distortion theorems, closure theorems, radii of close-to-convexity, starlikeness, and convexity for functions belonging to the class $\mathcal{U}_{\mathcal{T}}(\Phi, \Psi ; \alpha, \beta)$ are obtained. We also determine integral operators for functions in this class and some properties involving modified Hadamard products of several functions belonging to the class $\mathcal{U}_{\mathcal{T}}^{*}(\Phi, \Psi, \alpha, \beta)$.
\end{abstract}

\section{Introduction and Definitions}

Let $\mathcal{A}$ denote the class of functions of the form :

$$
f(z)=z+\sum_{n=2}^{\infty} a_{n} z^{n}
$$

which are analytic in the open unit disc $\Delta=\{z:|z|<1\}$. Further, let $\mathcal{S}$ denote the subclass of $\mathcal{A}$ consisting of univalent functions in $\Delta$. We define the family $\mathcal{U}(\Phi, \Psi ; \alpha, k)$ consisting of the functions $f \in \mathcal{A}$ so that

$$
\operatorname{Re}\left\{\frac{f(z) * \Phi(z)}{f(z) * \Psi(z)}-\alpha\right\}>k\left|\frac{f(z) * \Phi(z)}{f(z) * \Psi(z)}-1\right|, \quad(z \in \Delta)
$$

where $-1 \leq \alpha<1, k \geq 0, \Phi(z)=z+\sum_{n=2}^{\infty} \lambda_{n} z^{n}$ and $\Psi(z)=z+\sum_{n=2}^{\infty} \mu_{n} z^{n}$ are analytic in $\Delta$ with the conditions $\lambda_{n} \geq 0, \mu_{n} \geq 0, \lambda_{n} \geq \mu_{n}$ for $n \geq 2$, and $f(z) * \Psi(z) \neq 0$.

The operator "*" stands for the Hadamard product or convolution of two power series $f(z)=z+\sum_{n=2}^{\infty} a_{n} z^{n}$ and $g(z)=z+\sum_{n=2}^{\infty} c_{n} z^{n}$ given by $f(z) * g(z)=z+\sum_{n=2}^{\infty} a_{n} c_{n} z^{n}$.

The family $\mathcal{U}(\Phi, \Psi ; \alpha, k)$ is of special interest because it reduces to various classes of well-known functions as well as many new ones. For example

$$
\mathcal{U}\left(\frac{z}{(1-z)^{2}}, \frac{z}{1-z} ; 0, k\right)=k-\mathcal{S} \mathcal{T} \equiv \operatorname{Re}\left(\frac{z f^{\prime}(z)}{f(z)}\right)>k\left|\frac{z f^{\prime}(z)}{f(z)}-1\right|
$$

Received May 20, 2004; revised December 2, 2004.

2000 Mathematics Subject Classification. 30C45.

Key words and phrases. Analytic and univalent functions, Hadamard product, uniformly convex and uniformly starlike functions. 
and

$$
\mathcal{U}\left(\frac{z+z^{2}}{(1-z)^{3}}, \frac{z}{(1-z)^{2}} ; 0, k\right)=k-\mathcal{U C V} \equiv \operatorname{Re}\left(1+\frac{z f^{\prime \prime}(z)}{f^{\prime}(z)}\right)>k\left|\frac{z f^{\prime \prime}(z)}{f^{\prime}(z)}\right| .
$$

are, respectively, the subclasses of $\mathcal{S}$ consisting of functions which are $k$ - starlike and $k$-uniformly convex in $\Delta$ introduced by Kanas and Winsiowska [1,2] ( see also the work of Kanas and Srivastava [3] and Gangadharan et al.[4]). In particular, when $k=1$, we obtain $1-\mathcal{S} \mathcal{T} \equiv \mathcal{S P}$ and $1-\mathcal{U C V} \equiv \mathcal{U C V}$, where $\mathcal{S P}$ and $\mathcal{U C V}$ are the familiar classes of uniformly convex functions and parabolic starlike functions in $\Delta$, respectively (see, for details, [5-8]). Another subclass is the subclass

$$
\mathcal{U}\left(\frac{z+z^{2}}{(1-z)^{3}}, \frac{z}{(1-z)^{2}} ; \alpha, 1\right)=\mathcal{U C V}(\alpha) \equiv \operatorname{Re}\left(1+\frac{z f^{\prime \prime}(z)}{f^{\prime}(z)}\right)-\alpha>k\left|\frac{z f^{\prime \prime}(z)}{f^{\prime}(z)}\right|
$$

of uniformly convex functions of order $\alpha$ which is defined by Rønning [9].

Let $\mathcal{T}$ denotes the subclass of $\mathcal{S}$ consisting of functions of the form:

$$
f(z)=z-\sum_{n=2}^{\infty} a_{n} z^{n} \quad\left(a_{n} \geq 0\right)
$$

Further, let

$$
\mathcal{U}_{\mathcal{T}}(\Phi, \Psi ; \alpha, \beta)=\mathcal{U}(\Phi, \Psi, \alpha, \beta) \cap \mathcal{T}
$$

In the present paper, we prove various coefficient inequalities, distortion theorems, closure theorems, radii of close-to-convexity, starlikeness, and convexity for functions belonging to the class $\mathcal{U}_{\mathcal{T}}(\Phi, \Psi ; \alpha, k)$. We also determine some properties involving modified Hadamard products and integral operator of several functions in this class.

\section{Coefficient Inequalities}

Theorem 1. Given $\alpha(1 \leq \alpha<1), k \geq 0$. If

$$
\sum_{n=2}^{\infty}\left[(1+k) \lambda_{n}-(\alpha+k) \mu_{n}\right]\left|a_{n}\right| \leq 1-\alpha
$$

then $f(z) \in \mathcal{U}(\Phi, \Psi ; \alpha, k)$.

Proof. It suffices to show that

$$
k\left|\frac{f(z) * \Phi(z)}{f(z) * \Psi(z)}-1\right|-\operatorname{Re}\left\{\frac{f(z) * \Phi(z)}{f(z) * \Psi(z)}-1\right\} \leq 1-\alpha
$$

We have

$$
\begin{aligned}
& k\left|\frac{f(z) * \Phi(z)}{f(z) * \Psi(z)}-1\right|-\operatorname{Re}\left\{\frac{f(z) * \Phi(z)}{f(z) * \Psi(z)}-1\right\} \leq(1+k)\left|\frac{f(z) * \Phi(z)}{f(z) * \Psi(z)}-1\right| \\
\leq & \frac{(1+k) \sum_{n=2}^{\infty}\left(\lambda_{n}-\mu_{n}\right)\left|a_{n}\right||z|^{n}}{1-\sum_{n=2}^{\infty} \mu_{n}\left|a_{n}\right||z|^{n}} \leq \frac{(1+k) \sum_{n=2}^{\infty}\left(\lambda_{n}-\mu_{n}\right)\left|a_{n}\right|}{1-\sum_{n=2}^{\infty} \mu_{n}\left|a_{n}\right|} .
\end{aligned}
$$


The last expression is bounded above by $1-\alpha$ if

$$
\sum_{n=2}^{\infty}\left[(1+k) \lambda_{n}-(\alpha+k) \mu_{n}\right]\left|a_{n}\right| \leq(1-\alpha)
$$

which is equivalent to $(2.1)$.

Now we prove that the condition (2.1) is also necessary for $f(z) \in \mathcal{U}_{\mathcal{T}}(\Phi, \Psi ; \alpha, k)$.

Theorem 2. $f(z) \in \mathcal{U}_{\mathcal{T}}(\Phi, \Psi, \alpha, k)$ for $\alpha(-1 \leq \alpha<1)$ and $k \geq 0$, iff

$$
\sum_{n=2}^{\infty}\left[(1+k) \lambda_{n}-(\alpha+k) \mu_{n}\right] a_{n} \leq 1-\alpha .
$$

The result (2.2) is sharp.

Proof. In view of Theorem 1, we need only to prove the necessity. Let $f(z) \in$ $\mathcal{U}_{\mathcal{T}}(\Phi, \Psi ; \alpha, k)$ and $z$ is real then

$$
\frac{1-\sum_{n=2}^{\infty} \lambda_{n} a_{n} z^{n-1}}{1-\sum_{n=2}^{\infty} \mu_{n} a_{n} z^{n-1}}-\alpha \geq \frac{1-\sum_{n=2}^{\infty}\left(\lambda_{n}-\mu_{n}\right) a_{n} z^{n-1}}{1-\sum_{n=2}^{\infty} \mu_{n} a_{n} z^{n-1}}
$$

Letting $z \rightarrow 1$ along the real axis, we obtain the inequality (2.2).

The result (2.2) is sharp for the function

$$
f(z)=z-\frac{1-\alpha}{(1+\beta) \lambda_{n}-(\alpha+\beta) \mu_{n}} z^{n} \quad(n \geq 2) .
$$

For the notational convenience we shall henceforth denote

$$
\sigma_{n}(\alpha, k)=(1+k) \lambda_{n}-(\alpha+k) \mu_{n} \quad(n \geq 2)
$$

\section{Growth and Distortion Theorems}

Theorem 3. Let the function $f(z) \in \mathcal{U}_{\mathcal{T}}(\Phi, \Psi ; \alpha, k)$. If $\left\{\sigma_{n}(\alpha, k)\right\}_{n=2}^{\infty}$ is a nondecreasing sequence, then, for $|z|=r<1$

$$
r-\frac{1-\alpha}{\sigma_{2}(\alpha, k)} r^{2} \leq|f(z)| \leq r+\frac{1-\alpha}{\sigma_{2}(\alpha, k)} r^{2}
$$

and if $\left\{\sigma_{n}(\alpha, k) / n\right\}_{n=2}^{\infty}$ is a non-decreasing sequence, then, for $|z|=r<1$

$$
1-\frac{2(1-\alpha)}{\sigma_{2}(\alpha, \beta)} r \leq\left|f^{\prime}(z)\right| \leq 1+\frac{2(1-\alpha)}{\sigma_{2}(\alpha, \beta)} r .
$$


The results (3.1) and (3.2) are sharp for the function $f(z)$ given

$$
f(z)=z-\frac{1-\alpha}{\sigma_{2}(\alpha, k)} z^{2} \quad(z= \pm r) .
$$

Proof. In view of Theorem 2, we note that $\sigma_{2}(\alpha, k) \sum_{n=2}^{\infty} a_{n} \leq \sum_{n=2}^{\infty} \sigma_{n}(\alpha, k) a_{n} \leq 1-\alpha$. Thus

$$
|f(z)| \geq|z|-\sum_{n=2}^{\infty} a_{n}|z|^{n} \geq r-r^{2} \sum_{n=2}^{\infty} a_{n} \geq r-\frac{1-\alpha}{\sigma_{2}(\alpha, k)} r^{2}
$$

Similarly,

$$
|f(z)| \leq|z|+\sum_{n=2}^{\infty} a_{n}|z|^{n} \leq r+r^{2} \sum_{n=2}^{\infty} a_{n} \leq r+\frac{1-\alpha}{\sigma_{2}(\alpha, k)} r^{2} .
$$

Also from Theorem 2, we have $\frac{\sigma_{2}(\alpha, \beta)}{2} \sum_{n=2}^{\infty} n a_{n} \leq \sum_{n=2}^{\infty} n \sigma_{n}(\alpha, \beta) a_{n} \leq 1-\alpha$. Thus

$$
\left|f^{\prime}(z)\right| \geq 1-\sum_{n=2}^{\infty} n a_{n}|z|^{n-1} \geq 1-r \sum_{n=2}^{\infty} n a_{n} \geq 1-\frac{2(1-\alpha)}{\sigma_{2}(\alpha, k)} r .
$$

On the other hand,

$$
\left|f^{\prime}(z)\right| \leq 1+\sum_{n=2}^{\infty} n a_{n}|z|^{n-1} \leq 1+r \sum_{n=2}^{\infty} n a_{n} \leq 1+\frac{2(1-\alpha)}{\sigma_{2}(\alpha, k)} r
$$

This completes the proof.

Corollary 1. The disk $|z|<1$ is mapped onto a domain that contains the disk $|w|<\frac{\sigma_{2}(\alpha, k)-(1-\alpha)}{\sigma_{2}(\alpha, k)}$ by any $f(z) \in \mathcal{U}_{\mathcal{T}}(\Phi, \Psi ; \alpha, k)$. The theorem is sharp with extermal function $f(z)$ given by (3.3).

Proof. The proof follow upon letting $r \rightarrow 1$ in (3.1).

\section{Closure Theorems}

In this section, we shall prove that the class $\mathcal{U}_{\mathcal{T}}(\Phi, \Psi ; \alpha, k)$ is closed under convex linear combinations.

Theorem 4. Let the function $f_{i}(z), i=1,2, \ldots, m$, defined by

$$
f_{i}(z)=z-\sum_{n=2}^{\infty} a_{n, i} z^{n} \quad\left(a_{n, i} \geq 0\right)
$$


for $z \in \Delta$, be in the class $\mathcal{U}_{\mathcal{T}}(\Phi, \Psi ; \alpha, k)$. Then the function $h(z)$ defined by

$$
h(z)=z-\sum_{n=2}^{\infty}\left(\frac{1}{m} \sum_{i=1}^{m} a_{n, i}\right) z^{n}
$$

also belongs to the class $\mathcal{U}_{\mathcal{T}}(\Phi, \Psi, \alpha, k)$.

Proof. Let $f_{i}(z) \in \mathcal{U}_{\mathcal{T}}(\Phi, \Psi ; \alpha, k)$,it follows from Theorem 2 that

$$
\sum_{n=2}^{\infty} \sigma_{n}(\alpha, k) a_{n, i} \leq 1-\alpha \quad(i=1,2, \ldots, m) .
$$

Therefore,

$$
\begin{aligned}
& \sum_{n=2}^{\infty} \sigma_{n}(\alpha, k)\left(\frac{1}{m} \sum_{i=1}^{m} a_{n, i}\right) \\
= & \frac{1}{m} \sum_{i=1}^{m}\left(\sum_{n=2}^{\infty} \sigma_{n}(\alpha, k) a_{n, i}\right) \leq 1-\alpha .
\end{aligned}
$$

Hence by Theorem $2, h(z) \in \mathcal{U}_{\mathcal{T}}(\Phi, \Psi ; \alpha, k)$.

With the aid of Theorem 2, we can prove the following

Theorem 5. Let the functions $f_{i}(z)$ be defined by (4.1) be in the class $\mathcal{U}_{\mathcal{T}}(\Phi, \Psi, \alpha, k)$ for every $i=1,2, \ldots, m$. Then the functions

$$
h(z)=\sum_{i=1}^{m} c_{i} f_{i}(z) \quad\left(c_{i} \geq 0\right)
$$

is also in the same class $\mathcal{U}_{\mathcal{T}}(\Phi, \Psi, \alpha, k)$ where $\sum_{i=1}^{m} c_{i}=1$.

Theorem 6. Let $f_{1}(z)=z$ and

$$
f_{n}(z)=z-\frac{1-\alpha}{\sigma_{n}(\alpha, k)} z^{n} \quad(n \geq 2) .
$$

Then $f(z) \in \mathcal{U}_{\mathcal{T}}(\Phi, \Psi ; \alpha, k)$ if and only if it can be expressed in the form:

$$
f(z)=\sum_{n=1}^{\infty} \delta_{n} f_{n}(z)
$$

where

$$
\delta_{n} \geq 0 \quad(n \geq 1) \text { and } \sum_{n=1}^{\infty} \delta_{n}=1
$$


Proof. Assume that

$$
f(z)=\sum_{n=1}^{\infty} \delta_{n} f_{n}(z)=z-\sum_{n=2}^{\infty} \frac{1-\alpha}{\sigma_{n}(\alpha, k)} \delta_{n} z^{n} .
$$

Then it follows that

$$
\sum_{n=2}^{\infty} \frac{\sigma_{n}(\alpha, k)}{1-\alpha} \cdot \frac{1-\alpha}{\sigma_{n}(\alpha, k)} \delta_{n}=\sum_{n=2}^{\infty} \delta_{n}=1-\delta_{1} \leq 1 .
$$

So by Theorem $1, f(z) \in \mathcal{U}_{\mathcal{T}}(\Phi, \Psi ; \alpha, k)$.

Conversely, assume that the function $f(z) \in \mathcal{U}_{\mathcal{T}}(\Phi, \Psi ; \alpha, k)$. Then

$$
a_{n} \leq \frac{1-\alpha}{\sigma_{n}(\alpha, k)} \quad(n \geq 2)
$$

Setting

$$
\delta_{n}=\frac{\sigma_{n}(\alpha, k)}{1-\alpha} a_{n} \quad(n \geq 2)
$$

and

$$
\delta_{1}=1-\sum_{n=2}^{\infty} \delta_{n}
$$

we can see that $f(z)$ can be expressed in the form (4.8). This completes the proof of the Theorem 6 .

\section{Radii of Close-to-convexity, Starlikeness and Convexity}

Theorem 7. Let the function $f(z) \in \mathcal{U}_{\mathcal{T}}(\Phi, \Psi ; \alpha, k)$. Then $f(z)$ is close-to-convex of order $\rho(0 \leq \rho<1)$ in $|z|<r_{1}$, where

$$
r_{1}=\inf _{n}\left[\frac{\sigma_{n}(\alpha, k)(1-\rho)}{n(1-\alpha)}\right]^{1 /(n-1)} \quad(n \geq 2) .
$$

The result is sharp, the extermal function $f(z)$ being given by (2.3).

Proof. Let $f(z) \in \mathcal{U}_{\mathcal{T}}(\Phi, \Psi ; \alpha, k)$, then the function $f(z)$ defined by (1.3) is close-toconvex of order $\rho$ in $|z|<r_{1}$, provided that

$$
\begin{aligned}
\left|f^{\prime}(z)-1\right| & =\left|\sum_{n=2}^{\infty} n a_{n} z^{n-1}\right| \\
& \leq \sum_{n=2}^{\infty} n a_{n}|z|^{n-1} \leq 1-\rho, \quad\left(|z|<r_{1} ; 0 \leq \rho<1\right)
\end{aligned}
$$


where $r_{1}$ is given by (5.1). But, by Theorem 2, (5.2) will be true if

$$
\left(\frac{n}{1-\rho}\right)|z|^{n-1} \leq \frac{\sigma_{n}(\alpha, k)}{1-\alpha}
$$

that is, if

$$
|z| \leq\left[\frac{\sigma_{n}(\alpha, k)(1-\rho)}{n(1-\alpha)}\right]^{1 /(n-1)} \quad(n \geq 2) .
$$

Theorem 7 follows easily from (5.3).

Theorem 8. Let the function $f(z) \in \mathcal{U}_{\mathcal{T}}(\Phi, \Psi ; \alpha, k)$. Then $f(z)$ is starlike of order $\rho(0 \leq \rho<1)$ in $|z|<r_{2}$, where

$$
r_{2}=\inf _{n}\left[\frac{\sigma_{n}(\alpha, k)(1-\rho)}{(n-\rho)(1-\alpha)}\right]^{1 /(n-1)} \quad(n \geq 2) .
$$

The result is sharp, with the extermal function $f(z)$ given by (2.3).

Proof. It is sufficient to show that $\left|\frac{z f^{\prime}(z)}{f(z)}-1\right| \leq 1-\rho$ for $|z|<r_{2}$, where $r_{2}$ is given by (5.4). From (1.3) we find that

$$
\left|\frac{z f^{\prime}(z)}{f(z)}-1\right| \leq \frac{\sum_{n=2}^{\infty}(n-1) a_{n}|z|^{n-1}}{1-\sum_{n=2}^{\infty} a_{n}|z|^{n-1}} .
$$

Thus $\left|\frac{z f^{\prime}(z)}{f(z)}-1\right| \leq 1-\rho$ if

$$
\sum_{n=2}^{\infty}\left(\frac{n-\rho}{1-\rho}\right) a_{n}|z|^{n-1} \leq 1
$$

But, by Theorem 2, (5.5) will be true if

$$
\left(\frac{n-\rho}{1-\rho}\right)|z|^{n-1} \leq \frac{\sigma_{n}(\alpha, k)}{1-\alpha}
$$

that is, if

$$
|z| \leq\left[\frac{\sigma_{n}(\alpha, k)(1-\rho)}{(n-\rho)(1-\alpha)}\right]^{1 /(n-1)} \quad(n \geq 2) .
$$

Theorem 8 follows easily from (5.6).

Corollary 2. Let the function $f(z) \in \mathcal{U}_{\mathcal{T}}(\Phi, \Psi ; \alpha, k)$. Then $f(z)$ is convex of order $\rho(0 \leq \rho<1)$ in $|z|<r_{3}$, where

$$
r_{3}=\inf _{n}\left[\frac{\sigma_{n}(\alpha, k)(1-\rho)}{n(n-\rho)(1-\alpha)}\right]^{1 /(n-1)} \quad(n \geq 2) .
$$


The result is sharp, with the extermal function $f(z)$ given by (2.3).

\section{Integral Operators}

Theorem 9. Let the functions $f(z)$ defined by $(1.3)$ be in the class $\mathcal{U}_{\mathcal{T}}(\Phi, \Psi ; \alpha, k)$, and let $c$ be real number such that $c>-1$. Then the function $F(z) \in \mathcal{U}_{\mathcal{T}}(\Phi, \Psi ; \alpha, k)$, where

$$
F(z)=\frac{c+1}{z^{c}} \int_{0}^{z} t^{c-1} f(t) d t
$$

Proof. From (6.1), it follows that

$$
F(z)=z-\sum_{n=2}^{\infty}\left(\frac{c+1}{c+n}\right) a_{n} z^{n}
$$

Therefore,

$$
\sum_{n=2}^{\infty}\left(\frac{\sigma_{n}(\alpha, k)}{1-\alpha}\right)\left(\frac{c}{n+c+1}\right) a_{n} \leq \sum_{n=2}^{\infty}\left(\frac{\sigma_{n}(\alpha, k)}{1-\alpha}\right) a_{n} \leq 1
$$

since $f(z) \in \mathcal{U}_{\mathcal{T}}(\Phi, \Psi ; \alpha, k)$. Hence, by Theorem $2, F(z) \in \mathcal{U}_{\mathcal{T}}(\Phi, \Psi ; \alpha, k)$

Theorem 10. Let the function $F(z)=z-\sum_{n=2}^{\infty} a_{n} z^{n}\left(a_{n} \geq 0\right)$ be in the class $\mathcal{U}_{\mathcal{T}}(\Phi, \Psi ; \alpha, k)$ and let $c$ be a real number such that $c>-1$. Then the function given by (6.1) is univalent in $|z|<r_{4}$, where

$$
r_{4}=\inf _{n}\left[\frac{\sigma_{n}(\alpha, k)(c+1)}{n(c+n)|\beta|}\right]^{1 /(n-1)} \quad(n \geq 2) .
$$

The result is sharp.

Proof. From (6.1), we have

$$
f(z)=\frac{z^{1-c}\left(z^{c} F(z)\right)^{\prime}}{c+1}=z-\sum_{n=2}^{\infty}\left(\frac{c+n}{c+1}\right) a_{n} z^{n} .
$$

In order to obtain the required result, it suffices to show that $\left|f^{\prime}(z)-1\right|<1$ whenever $|z|<r_{4}$, where $r_{4}$ is given by (6.2). Now

$$
\left|f^{\prime}(z)-1\right| \leq \sum_{n=2}^{\infty} n\left(\frac{c+n}{c+1}\right) a_{n}|z|^{n-1} .
$$

Thus $\left|f^{\prime}(z)-1\right|<1$ if

$$
\sum_{n=2}^{\infty} n\left(\frac{c+n}{c+1}\right) a_{n}|z|^{n-1}<1
$$


But from Theorem 2, (6.3) will be satisfied if

$$
\frac{n(c+n)}{c+1}|z|^{n-1}<\frac{\sigma_{n}(\alpha, k)}{1-\alpha}
$$

that is, if

$$
|z| \leq\left[\frac{\sigma_{n}(\alpha, k)(c+1)}{n(c+n)(1-\alpha)}\right]^{1 /(n-1)} \quad(n \geq 2) .
$$

Therefore, $f(z)$ is univalent in $|z|<r_{4}$.

The result is sharp for the function

$$
f(z)=z-\frac{(c+n)(1-\alpha)}{\sigma_{n}(\alpha, k)(c+1)} z^{n} \quad(n \geq 2) .
$$

\section{Modified Hadamard Product}

Let the functions $f_{i}(z)(i=1,2)$ be defined by (4.1), then we define the modified Hadamard product of $f_{1}(z)$ and $f_{2}(z)$ by

$$
\left(f_{1} * f_{2}\right)(z)=z-\sum_{n=2}^{\infty} a_{n, 1} a_{n, 2} z^{n} .
$$

Employing the technique used earlier by Schild and Silverman [10], we prove the following

Theorem 11. Let each of the functions $f_{j}(z)(j=1,2)$ defined by (4.1) be in the class $\mathcal{U}_{\mathcal{T}}(\Phi, \Psi ; \alpha, k)$. Let

$$
\Delta(n)=\frac{\left(\sigma_{n}(\alpha, k)\right)^{2}-(1-\alpha)^{2}\left[(1+k) \lambda_{n}-k \mu_{n}\right]}{\left(\sigma_{n}(\alpha, k)\right)^{2}-(1-\alpha)^{2} \mu_{n}},
$$

If $\Delta(n)$ is an increasing function of $n(n \geq 2)$, then $\left(f_{1} * f_{2}\right)(z) \in \mathcal{U}_{\mathcal{T}}(\Phi, \Psi, \gamma, k)$, for

$$
\gamma=\frac{\left(\sigma_{2}(\alpha, k)\right)^{2}-(1-\alpha)^{2}\left[(1+k) \lambda_{2}-k \mu_{2}\right]}{\left(\sigma_{2}(\alpha, k)\right)^{2}-(1-\alpha)^{2} \mu_{2}} .
$$

The result is sharp.

Proof. We need to find the largest $\gamma$ such that

$$
\sum_{n=2}^{\infty} \frac{\sigma_{n}(\gamma, k)}{1-\gamma} a_{n, 1} a_{n, 2} \leq 1
$$


From Theorem 2, we have $\sum_{n=2}^{\infty} \frac{\sigma_{n}(\alpha, k)}{1-\alpha} a_{n, 1} \leq 1$ and $\sum_{n=2}^{\infty} \frac{\sigma_{n}(\alpha, k)}{1-\alpha} a_{n, 2} \leq 1$, by the CauchySchwarz inequality, we have

$$
\sum_{n=2}^{\infty} \frac{\sigma_{n}(\alpha, k)}{1-\alpha} \sqrt{a_{n, 1} a_{n, 2}} \leq 1 .
$$

Thus it is sufficient to show that

$$
\frac{\sigma_{n}(\gamma, k)}{1-\gamma} a_{n, 1} a_{n, 2} \leq \frac{\sigma_{n}(\alpha, k)}{1-\alpha} \sqrt{a_{n, 1} a_{n, 2}}, \quad(n \geq 2)
$$

that is, that

$$
\sqrt{a_{n, 1} a_{n, 2}} \leq \frac{\sigma_{n}(\alpha, k)(1-\gamma)}{\sigma_{n}(\gamma, k)(1-\alpha)} \quad(n \geq 2)
$$

Note that

$$
\sqrt{a_{n, 1} a_{n, 2}} \leq \frac{1-\alpha}{\sigma_{n}(\alpha, k)} \quad(n \geq 2) .
$$

Consequently, we need only to prove that

$$
\frac{1-\alpha}{\sigma_{n}(\alpha, k)} \leq \frac{\sigma_{n}(\alpha, k)(1-\gamma)}{\sigma_{n}(\gamma, k)(1-\alpha)} \quad(n \geq 2)
$$

or, equivalently

$$
\gamma \leq \frac{\left(\sigma_{n}(\alpha, k)\right)^{2}-(1-\alpha)^{2}\left[(1+k) \lambda_{n}-k \mu_{n}\right]}{\left(\sigma_{n}(\alpha, k)\right)^{2}-(1-\alpha)^{2} \mu_{n}}=\Delta(n)
$$

Since $\Delta(n)$ is an increasing function of $n(n \geq 2)$, letting $n=2$ in (7.4), we obtain

$$
\gamma \leq \Delta(2)=\frac{\left(\sigma_{2}(\alpha, k)\right)^{2}-(1-\alpha)^{2}\left[(1+k) \lambda_{2}-k \mu_{2}\right]}{\left(\sigma_{2}(\alpha, k)\right)^{2}-(1-\alpha)^{2} \mu_{2}},
$$

which proves the main assertion of Theorem 12 .

Finally, by taking the functions

$$
f_{j}(z)=z-\frac{1-\alpha}{\sigma_{2}(\alpha, k)} z^{2} \quad(j=1,2)
$$

we can see the result is sharp.

Theorem 12. Let each of the functions $f_{j}(z)(j=1,2)$ defined by (4.1) be in the class $\mathcal{U}_{\mathcal{T}}(\Phi, \Psi ; \alpha, k)$. Let

$$
\Omega(n)=\frac{\frac{1}{2}\left(\sigma_{n}(\alpha, k)\right)^{2}-\left[(1+k) \lambda_{n}-k \mu_{n}\right](1-\alpha)^{2}}{\frac{1}{2}\left(\sigma_{n}(\alpha, k)\right)^{2}-\mu_{n}(1-\alpha)^{2}},
$$


If $\Omega(n)$ is an increasing function of $n(n \geq 2)$, then the function

$$
h(z)=z-\sum_{n=2}^{\infty}\left(a_{n, 1}^{2}+a_{n, 2}^{2}\right) z^{n}
$$

belongs to the class $\mathcal{U}_{\mathcal{T}}(\Phi, \Psi ; \tau, k)$, where

$$
\tau=\frac{\frac{1}{2}\left(\sigma_{2}(\alpha, k)\right)^{2}-\left[(1+k) \lambda_{2}-k \mu_{2}\right](1-\alpha)^{2}}{\frac{1}{2}\left(\sigma_{2}(\alpha, k)\right)^{2}-\mu_{2}(1-\alpha)^{2}} .
$$

The result is sharp for the functions $f_{j}(z)(j=1,2)$ defined by $(7.5)$.

Proof. From Theorem 1, we have

$$
\sum_{n=2}^{\infty}\left[\frac{\sigma_{n}(\alpha, k)}{1-\alpha}\right]^{2} a_{n, 1}^{2} \leq \sum_{n=2}^{\infty}\left[\frac{\sigma_{n}(\alpha, k)}{1-\alpha} a_{n, 1}\right]^{2} \leq 1
$$

and

$$
\sum_{n=2}^{\infty}\left[\frac{\sigma_{n}(\alpha, \beta)}{1-\alpha}\right]^{2} a_{n, 2}^{2} \leq \sum_{k=2}^{\infty}\left[\frac{\sigma_{n}(\alpha, \beta)}{1-\alpha} a_{n, 2}\right]^{2} \leq 1 .
$$

It follows from (7.9) and (7.10) that

$$
\sum_{k=2}^{\infty} \frac{1}{2}\left[\frac{\sigma_{n}(\alpha, k)}{1-\alpha}\right]^{2}\left(a_{n, 1}^{2}+a_{n, 2}^{2}\right) \leq 1
$$

Therefore, we need to find the largest $\tau$ such that

$$
\frac{\sigma_{n}(\tau, k)}{1-\tau} \leq \frac{1}{2}\left[\frac{\sigma_{n}(\alpha, k)}{1-\alpha}\right]^{2} \quad(n \geq 2)
$$

that is,

$$
\tau \leq \frac{\frac{1}{2}\left(\sigma_{n}(\alpha, k)\right)^{2}-\left[(1+k) \lambda_{n}-k \mu_{n}\right](1-\alpha)^{2}}{\frac{1}{2}\left(\sigma_{n}(\alpha, k)\right)^{2}-\mu_{n}(1-\alpha)^{2}}=\Omega(n) .
$$

Since $\Omega(n)$ is an increasing function of $n(n \geq 2)$, we readily have

$$
\tau \leq \Omega(2)=\frac{\frac{1}{2}\left(\sigma_{2}(\alpha, k)\right)^{2}-\left[(1+k) \lambda_{2}-k \mu_{2}\right](1-\alpha)^{2}}{\frac{1}{2}\left(\sigma_{2}(\alpha, k)\right)^{2}-\mu_{2}(1-\alpha)^{2}} .
$$

Hence the result.

\section{References}

[1] S. Kanas and A. Wisniowska, Conic domains and k-starlike functions, Rev. Roumaine Math. Pures Appl. 45(2000), 647-657. 
[2] S. Kanas and A. Wisniowska, Conic regions and k-uniform convexity, J. Comput. App. Math. 105 (1999), 327-336.

[3] S. Kanas and H.M. Srivastava, Linear operators associated with k-uniformly convex functions, Integral Transform. Spec. Funct. 9 (2000), 121-132.

[4] A. Gangadharan, T.N. Shanmugan and H.M. Srivastava, Generalized hypergeometric functions associated with k-uniformaly convex functions, Comput. Math. App. 44 (2002), 1515-1526.

[5] A. W. Goodman, On uniformly convex functions, Ann. Polon. Math. 56 (1991), 87-92.

[6] A. W. Goodman, On uniformly starlike functions, J. Math. Anal. Appl. 155 (1991), 364-370

[7] W. C. Ma and D. Minda, Uniformly convex functions, Ann. Polon. Math. 57 (1992), $165-175$.

[8] F. Rønning, Uniformly convex functions and a corresponding class of starlike functions, Proc. Amer. Math. Soc. 118 (1993),189-196.

[9] F. Rønning, On starlike functions associated with parabolic regions, Ann Univ. Mariae Curie-Sklodowska Sect. A 45 (1991), 117-122.

[10] A. Schild and Silverman, Convolutions of univalent functions with negative coefficients, Ann. Univ. Mariae Curie-Scklodowska Sect. A 29 (1975), 99-107.

Department of Mathematics, Al al-Bayt University, P.O.Box: 130095 Mafraq, Jordan.

E-mail: bafrasin@yahoo.com 